Once the opening is controlled, it is easy to place a grasping instrument through the same working port as the triangular retractor to push the isolated lobe into the specimen extraction sac. Once its lead point is inserted, another instrument placed through the access incision maintains the specimen's forward progress so that the endoscopic grasper can gain a fresh hold on the tissue. With the cooperative use of these instruments, the lobe is inserted quickly. Figure 2 depicts the sac held open just after lung insertion.

\section{Discussion}

Thoracic surgeons periodically reevaluate their interests in complex VATS operations as technology advances. ${ }^{2}$ Recent enhancements in video equipment aid exposure, and new specialized tools reduce operative times, making these cases more relevant to a busy surgical practice.

Upper lobe tissue often flops onto the hilum. This requires a lot of camera shifting and lung rolling to ensure complete dissection and thereby prevent inadvertent avulsion of an aber- rant pulmonary artery branch. The described retraction method allows lifting the lung toward the lateral chest wall so that residual hilar connections can be viewed. Some authors advocate dividing the bronchus first to gain this exposure, but we prefer technology that allows us to approximate our traditional open methods.

Although introducing bulky lobes into specimen extraction sacs can be tedious, this technical modification reduced a variable 5- to 20-minute process into a routine step lasting 2 to 3 minutes. The study of other minimally invasive disciplines might yield additional useful instrument adaptations.

\section{References}

1. Swanson SJ, Herndon J, D’Amico TA, Demmy TL, McKenna R Jr, Green M, et al. Results of CALGB 39802: feasibility of video-assisted thoracic surgery (VATS) lobectomy for early stage lung cancer [abstract]. Proc Am Soc Clin Oncol. 2002;21:290a.

2. Demmy TL, Curtis JJ, Boley TM, Walls JT, Nawarawong W, Schmaltz RA. Diagnostic and therapeutic thoracoscopy: lessons from the learning curve. Am J Surg. 1993;166:696-700.

\title{
Successful subtotal tracheal replacement (using a skin/omental graft) for dehiscence after a resection for thyroid cancer
}

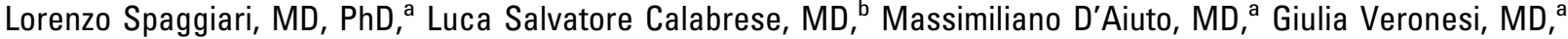 \\ Domenico Galetta, MD, ${ }^{a}$ Marco Venturino, MD, ${ }^{\mathrm{c}}$ and Fausto Chiesa, MD, ${ }^{\mathrm{b}}$ Milan, Italy
}

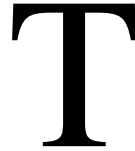
racheal resection is still one of the greatest challenges in the treatment of tracheal tumors. ${ }^{1}$ The maximum amount of trachea that can be resected during resection-anastomosis is around $50 \%$, but, when a larger resection is required, the risk of postoperative dehiscence increases, followed by a very elevated risk of postoperative death.

We report on our experience with treating a tracheal anastomotic dehiscence that developed after an extended tracheal resection was performed for a thyroid tumor relapse. The technique used to repair the dehiscence, a composite skin/omental/ muscle graft, permitted restoration of tracheal continuity and of a normal respiratory function.

\footnotetext{
From the Division of Thoracic Surgery, ${ }^{\mathrm{a}}$ Head and Neck Division, ${ }^{\mathrm{b}}$ and Division of Anesthesiology and Intensive Care Unit, ${ }^{\mathrm{c}}$ European Institute of Oncology, Milan, Italy.

Received for publication Oct 2, 2004; accepted for publication Nov 4, 2004.

Address for reprints: Lorenzo Spaggiari, MD, PhD, Division of Thoracic Surgery, European Institute of Oncology, Via Ripamonti, 435, 20141 Milan, Italy (E-mail: lorenzo.spaggiari@ieo.it).

J Thorac Cardiovasc Surg 2005; 129:1455-6

$0022-5223 / \$ 30.00$

Copyright $\odot 2005$ by The American Association for Thoracic Surgery

doi:10.1016/j.jtcvs.2004.11.010
}

\section{Clinical Summary}

In May 2001, a 37-year-old man underwent thyroidectomy for medullary carcinoma of the thyroid at another institution. In May 2002, he developed a relapse with tracheal infiltration and was admitted to our institute in July 2002 for an extended resection of the trachea. A double transmanubrial approach was used. $^{2}$ After an extensive tracheal devascularization, a $5.5-\mathrm{cm}$ long tracheal resection was performed. The tumor $(9.2 \mathrm{~cm}$ in diameter) was removed along with the trachea, thymus, and all cervical and mediastinal lymph nodes. Tracheal reconstruction was carried out by resection-anastomosis, in conjunction with tracheal release, the Dedo-Fishmann maneuver, and cervical flexion. Final diagnosis was medullary carcinoma of the thyroid pT4, pN1b. The patient was then immediately extubated, and, after 24 hours of intensive care unit stay, was returned to the hospital ward.

Six days after the operation, the patient developed a complete dehiscence of the tracheal suture, requiring emergency surgery. Due to the previous lateral devascularization, tracheal stumps appeared ischemic for about $2 \mathrm{~cm}$, resulting in an overall loss of about $9 \mathrm{~cm}$ of tracheal matter, leaving $1.5 \mathrm{~cm}$ of viable trachea above the carina and below the larynx, respectively. Tracheal continuity was reconstructed by grafting and suturing a $9 \times 6-\mathrm{cm}$ chest wall skin flap to the still viable distal and proximal tracheal stumps and, then, to the lateral esophageal margins; hence, the muscular wall of the esophagus gave 


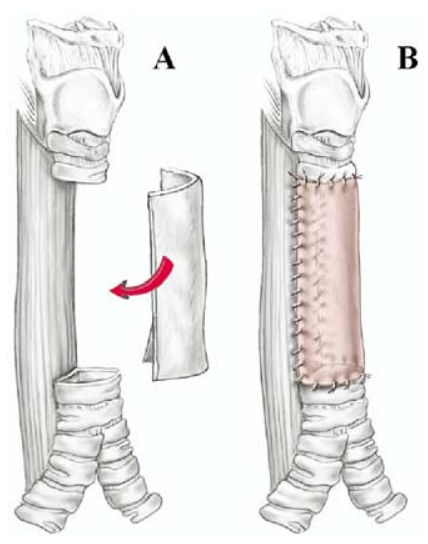

Figure 1. Schematic illustration of tracheal replacement. A, Tracheal segment, $9 \mathrm{~cm}$ in length, was removed. B, Termino-terminal skin graft-tracheal anastomosis was accomplished. Laterally, the skin graft was fixed to the lateral margins of the esophagus so that the anterior esophageal wall gave rise the membranous portion of the new trachea.

rise to the membranous portion of the new trachea (Figures 1 and 2). An omentoplasty was then used to cover up the graft. A myoplasty of the left major pectoral muscle onto the omentum completed the surgery, while resection of the manubrium and both the clavicular joints facilitated closing of the surgical wounds.

Postoperatively, the patient developed acute respiratory distress syndrome, requiring assisted ventilation for 21 days. Once spontaneous respiration was attained and before extubation was done, the patient was evaluated by a rigid bronchoscope, which showed a viable cutaneous flap without stenosis or mediastinal fistulas. Extubation was then done.

After 7 days, the patient began to demonstrate expiratory dyspnea. A bronchoscopic examination revealed the beginning of stenosis of the graft. An Ultraflex stent (Boston Scientific Corp, Natick, Mass) was then put in place, and a marked improvement in the clinical picture followed. Twenty-four months after the operation, the patient is doing well, with no recurrence.

\section{Discussion}

Even though numerous techniques have been proposed for restoring tracheal continuity after subtotal tracheal resection, ${ }^{1}$ end-to-end resection-anastomosis still remains the technique of choice. However, such a procedure is limited by the extent of resection; it cannot be performed on conditions necessitating resections greater than $50 \%$ because there is a high risk of early tracheal dehiscence. This occurrence is a serious complication, linked to a high risk of death. The development of such a

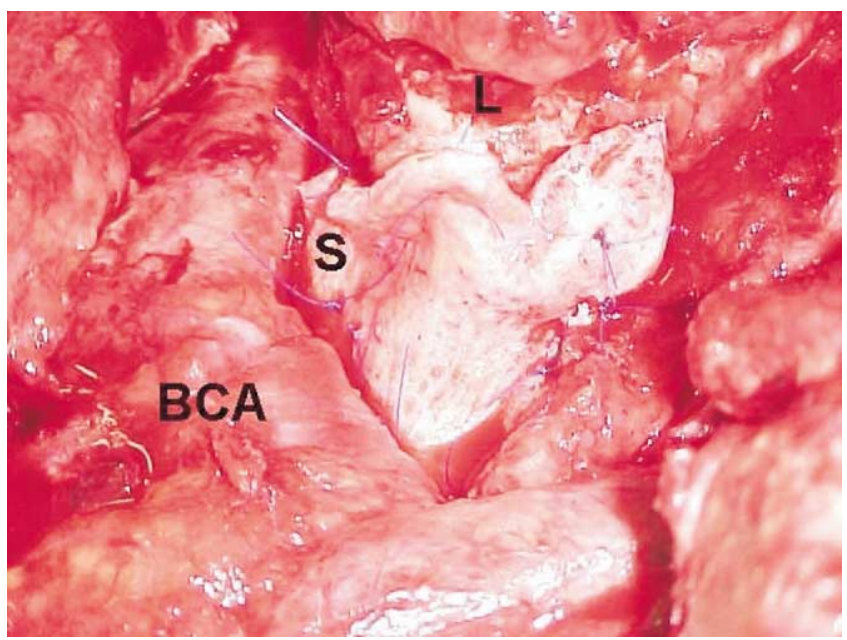

Figure 2. Operative field showing the completed tracheal replacement. $L$, Larynx; $S$, skin graft; $B C A$, brachiocephalic artery.

complication is multifactorial: Risk is associated with the length of the resection, the tension to which the suture is subjected after such a resection, and the extended lateral devascularization of the trachea. Previous treatments (chemotherapy/ radiotherapy) also significantly increase risk.

Skin and dermal grafts were tried in the past but with unsatisfactory results in terms of tracheal substitution. ${ }^{3}$ Yet, the addition of omentoplasty in conjunction with myoplasty allowed the graft to neovascularize quickly, keeping it viable, while the use of the esophagus as a back wall facilitated reconstruction of the trachea, reducing the size of the graft.

We believe that the key to success of this procedure was multifactorial: the omentum and the myoplasty used for vascularizing the graft; the "quiescence" of the graft as a result of the prolonged intubation, which facilitated cicatrization; the use of viable muscular wall of the esophagus as the integral part of the new trachea; and, lastly, the use of the endoscopic prosthesis to counteract the inevitable cicatricial stenosis.

Even though one "successful" case is not enough to validate a technique, the use of the skin/omentoplasty/myoplasty graft could prove beneficial in a dramatic situation like the one described here.

\section{References}

1. Grillo HC. Surgery of the trachea and bronchi. 1st ed. Hamilton: BC Decker Inc; 2004.

2. Spaggiari L, Pastorino U. Double transmanubrial approach and sternotomy for resection of a giant thymic carcinoid tumor. Ann Thorac Surg. 2001;72:629-31.

3. Grillo HC. Tracheal replacement. In: Grillo HC, editor. Surgery of the trachea and bronchi. 1st ed. Hamilton: BC Decker Inc; 2004. 